\title{
Heat current in a parametric quantum pump
}

\author{
Baigeng Wang ${ }^{1}$ and Jian Wang ${ }^{1,2, *}$ \\ ${ }^{1}$ Department of Physics, The University of Hong Kong, Pokfulam Road, Hong Kong, China \\ ${ }^{2}$ Institute of Solid State Physics, Chinese Academy of Sciences, Hefei, Anhui, China
}

(Received 3 April 2002; published 23 September 2002)

\begin{abstract}
We investigate the heat flow in the parametric quantum pump. Using the time dependent scattering matrix theory, we have developed a general theory for the pumped heat current at finite pumping amplitude and frequency. We have applied our theory to a double barrier structure and studied pumped heat current in both the weak and strong pumping regimes as different system parameters vary. By comparing the pumped heat current and the power of Joule heat generated in the system, we found that the double barrier structure can function as an optimal pump in the strong pumping regime.
\end{abstract}

DOI: 10.1103/PhysRevB.66.125310

PACS number(s): 73.23.Ad, 73.40.Gk, 72.10.Bg, 74.50.+r

The physics of adiabatic quantum pump has attracted great attention recently. ${ }^{1-18}$ The quantum pump is realized by varying the geometric parameters of the quantum dot, by which the current is generated. At the same time, heat current is also produced and accompanied with the dissipation. Avron et al. ${ }^{19}$ have given a lower bound for the dissipation in a quantum channel which is defined as the difference between the heat current and the power of Joule heat. The pump is optimal if the heat current equals the power of Joule heat. ${ }^{19}$ As a result, the optimal pump is noiseless and charge transported is quantized. In a recent paper, Moskalets and Buttiker $^{20}$ have also considered the dissipation in an adiabatic quantum pump. The heat current and the noise have been formulated in terms of a parametric emissivity matrix. The theory of Moskalets and Buttiker is in the weak pumping regime (quadratic order in pumping amplitude) and can go to the finite frequency if one goes beyond the hypothesis of instant scattering. In this paper, we develop a general theory for the heat current which is valid for finite pumping amplitude and finite frequency. This allows us to study the heat current in both weak and strong pumping regimes. Our theory is based on the time dependent scattering matrix theory ${ }^{20,8}$ and goes beyond the instant scattering hypothesis. We have applied our theory to a double barrier structure and studied pumped heat current as different system parameters such as Fermi energy, pumping amplitude, and phase difference vary. In the weak pumping regime, the pumped heat current increases quadratically as pumping amplitude increases. The dependence becomes linear in the strong pumping regime. In the strong pumping regime, the pumped heat current shows strong nonlineality as a function of phase difference between two pumping potentials. The heat current for single pump has also been studied. We found that the amplitude of the pumped heat current for the single pump is of the same order as that of two pumping potentials. Recently there has been a concern regarding the existence of a genuine optimal pump with nonvanishing transmission coefficient. $^{21}$ In this paper, we give a nontrivial example of optimal pump. By comparing the pumped heat current and the power of Joule heat generated in the system, we found that the double barrier structure is an optimal pump in the strong pumping regime.
We start with the general definition for the heat current in scattering matrix theory, ${ }^{20}$

$$
I_{q, \alpha}=\lim _{\Delta t \rightarrow \infty} \frac{1}{\Delta t} \int_{0}^{\Delta t} d t\left\langle\hat{I}_{q, \alpha}\right\rangle,
$$

where the heat current operator is $\hat{I}_{q, \alpha}=\hat{I}_{E, \alpha}-E_{F} \hat{I}_{e, \alpha}$ and $\langle\cdots\rangle$ denotes the quantum average. Here $\hat{I}_{E, \alpha}$ is the energy current operator given by

$$
\hat{I}_{E, \alpha}=-i\left[\partial_{t} \hat{b}_{\alpha}^{\dagger}(t) \hat{b}_{\alpha}(t)-\partial_{t} \hat{a}_{\alpha}^{\dagger}(t) \hat{a}_{\alpha}(t)\right]
$$

and $\hat{I}_{e, \alpha}$ is the electric current operator,

$$
\hat{I}_{e, \alpha}=\hat{b}_{\alpha}^{\dagger}(t) \hat{b}_{\alpha}(t)-\hat{a}_{\alpha}^{\dagger}(t) \hat{a}_{\alpha}(t),
$$

where the operators $\hat{b}_{\alpha}$ and $\hat{a}_{\alpha}$ are annihilation operators for the outgoing and incoming carriers in the lead $\alpha$. They are related by the scattering matrix,

$$
\hat{b}_{\alpha}(t)=\sum_{\beta} \int d t^{\prime} s_{\alpha \beta}\left(t, t^{\prime}\right) \hat{a}_{\beta}\left(t^{\prime}\right),
$$

where the time dependence of the scattering matrix is due to the slowly time-varying pumping potential $X(t)$. The distribution function can be obtained by taking the quantum average, ${ }^{20}$

$$
\left\langle\hat{a}_{\alpha}^{\dagger}(E) \hat{a}_{\beta}\left(E^{\prime}\right)\right\rangle=\delta_{\alpha \beta} \delta\left(E-E^{\prime}\right) f_{\alpha}(E),
$$

where $\hat{a}_{\alpha}(E)$ is the Fourier transform of $\hat{a}_{\alpha}(t)$ and $f(E)$ is the Fermi distribution function. For the purpose of presentation, we calculate the energy current first and the electric current can be calculated in a similar fashion. From Eqs. (2), (4), and (5), the energy current is given by

$$
\begin{aligned}
I_{E, \alpha}= & -\lim _{\Delta t \rightarrow \infty} \frac{i}{\Delta t} \int_{0}^{\Delta t} d t \int d t_{1} d t_{2} \sum_{\beta} s_{\alpha \beta}\left(t, t_{1}\right) \\
& \times f\left(t_{1}-t_{2}\right) \partial_{t} s_{\alpha \beta}^{\dagger}\left(t, t_{2}\right)-\int \frac{d E}{2 \pi} E f(E),
\end{aligned}
$$

where $f(t) \equiv \int(d E / 2 \pi) \exp (-i E t) f(E)$. Now we will focus on the first term (denoted as $I_{E, \alpha}^{(1)}$ ) in Eq. (6). After changing of the variable $t_{0}=\left(t_{1}+t_{2}\right) / 2$ and $\tau=t_{1}-t_{2}$ and using the following Wigner transform for the scattering matrix: ${ }^{8}$ 


$$
s\left(t, t^{\prime}\right)=\int \frac{d \epsilon}{2 \pi} e^{-i \epsilon\left(t-t^{\prime}\right)} s\left(\epsilon, \frac{t+t^{\prime}}{2}\right),
$$

Eq. (6) becomes,

$$
\begin{aligned}
I_{E, \alpha}^{(1)}= & \lim _{\Delta t \rightarrow \infty} \frac{-i}{4 \pi^{2} \Delta t} \int_{0}^{\Delta t} d t \int d t_{0} d \tau d \epsilon_{1} d \epsilon_{2} f(\tau) \\
& \times e^{-i \epsilon_{1}\left(t-t_{0}-\tau / 2\right)} e^{i \epsilon_{2}\left(t-t_{0}+\tau / 2\right)} \\
& \times \sum_{\beta} s_{\alpha \beta}\left(\epsilon_{1}, \frac{t+t_{0}}{2}+\frac{\tau}{4}\right)\left[\partial_{t} s_{\alpha \beta}^{\dagger}\left(\epsilon_{2}, \frac{t+t_{0}}{2}-\frac{\tau}{4}\right)\right. \\
& \left.+i \epsilon_{2} s_{\alpha \beta}^{\dagger}\left(\epsilon_{2}, \frac{t+t_{0}}{2}-\frac{\tau}{4}\right)\right] .
\end{aligned}
$$

Changing the variables again to $\tau_{1}=t-t_{0}$ and $t^{\prime}=(t$ $\left.+t_{0}\right) / 2$ and integrating over $\tau_{1}$, we obtain

$$
\begin{aligned}
I_{E, \alpha}^{(1)}= & \lim _{\Delta t \rightarrow \infty} \frac{-i}{2 \pi \Delta t} \int_{-\Delta t}^{\Delta t} d t^{\prime} d \tau d \epsilon \sum_{\beta} s_{\alpha \beta}\left(\epsilon, t^{\prime}+\tau / 4\right) f(\tau) \\
& \times\left[(1 / 2) \partial_{t^{\prime}} s_{\alpha \beta}^{\dagger}\left(\epsilon, t^{\prime}-\tau / 4\right)+i \epsilon s_{\alpha \beta}^{\dagger}\left(\epsilon, t^{\prime}-\tau / 4\right)\right] e^{i \epsilon \tau} .
\end{aligned}
$$

To get the heat current up to $\omega^{2}$, it is enough to expand $s_{\alpha \beta}$ up to the second order in $\tau$. We obtain

$$
\begin{aligned}
I_{E, \alpha}= & -\frac{1}{8 \pi T_{p}} \int_{0}^{T_{p}} d t \int d E\left[-E \partial_{E}^{2} f\right] \\
& \times \sum_{\beta} \partial_{t} s_{\alpha \beta}(E, t) \partial_{t} s_{\alpha \beta}^{\dagger}(E, t)-\frac{1}{2 \pi T_{p}} \\
& \times \int_{0}^{T_{p}} d t \int d E E \partial_{E} f \sum_{\beta} \operatorname{Im}\left[\partial_{t} s_{\alpha \beta}^{\dagger}(E, t) s_{\alpha \beta}(E, t)\right],
\end{aligned}
$$

where the zeroth order term in $\tau$ in Eq. (9) has been canceled by the second term in Eq. (6) and $T_{p}$ is the period of the pumping cycle. Including the electric current, the heat current is given by

$$
\begin{aligned}
I_{q, \alpha}= & -\frac{1}{8 \pi T_{p}} \int_{0}^{T_{p}} d t \int d E\left[-\left(E-E_{F}\right) \partial_{E}^{2} f\right] \\
& \times \sum_{\beta} \partial_{t} s_{\alpha \beta}(E, t) \partial_{t} s_{\alpha \beta}^{\dagger}(E, t)-\frac{1}{2 \pi T_{p}} \\
& \times \int_{0}^{T_{p}} d t \int d E\left(E-E_{F}\right) \partial_{E} f \\
& \times \sum_{\beta} \operatorname{Im}\left[\partial_{t} s_{\alpha \beta}^{\dagger}(E, t) s_{\alpha \beta}(E, t)\right] .
\end{aligned}
$$

Note that the second term in Eq. (11) vanishes at zero temperature. In the adiabatic regime, we have ${ }^{8} s_{\alpha \beta}$ $=\Sigma_{i}\left[\partial_{X_{i}} s_{\alpha \beta} \partial_{t} X_{i}+\partial_{\dot{X}_{i}} s_{\alpha \beta} \partial_{t} \dot{X}_{i}+\cdots\right]$ where $\dot{X} \equiv d X / d t$. Up to the order $\omega^{2}$, we can neglect the contribution from $\partial_{\dot{X}_{i}} s_{\alpha \beta}$. At zero temperature, Eq. (11) becomes,

$$
I_{q, \alpha}=\frac{1}{8 \pi T_{p}} \int_{0}^{T_{p}} d t \sum_{\beta} \sum_{i j} \partial_{X_{i}} S_{\alpha \beta} \partial_{X_{j}} S_{\alpha \beta}^{\dagger} \partial_{t} X_{i} \partial_{t} X_{j} .
$$

It is straightforward to obtain the heat current at higher order in frequency. To do that, we have to keep the higher-order term in the expansion of $\tau$ in Eq. (9) and include the contribution of higher-order derivatives ${ }^{3,8}$ such as $\dot{X}$. For instance, expanding Eq. (9) to the third order in $\tau$ and including the contribution from the electric current, we obtain (up to $\omega^{3}$ ),

$$
I_{q, \alpha}^{(3)}=\frac{1}{48 \pi T_{p}} \int_{0}^{T_{p}} d t \int d E \partial_{E}^{2} f \sum_{\beta} \operatorname{Im}\left[\partial_{t}^{2} s_{\alpha \beta} \partial_{t} s_{\alpha \beta}^{\dagger}\right] .
$$

There are also third order corrections from Eq. (11) where the term $\partial_{\dot{X}_{i}} S_{\alpha \beta}$ should be kept. We now consider the limiting case of Eq. (12) when the pumping amplitude is small. For two probe pumping: $X_{1}(t)=X_{1} \sin (\omega t)$ and $X_{2}(t)=X_{2} \sin (\omega t$ $+\phi)$, we see that the lowest order pumping amplitude in Eq. (12) is quadratic by neglecting $X$ dependence in $\partial_{X} s_{\alpha \beta}$. In this case, it is straightforward to show that Eq. (12) is reduced to

$$
\begin{aligned}
I_{q, \alpha}= & \frac{\omega^{2}}{16 \pi}\left[X_{1}^{2} \sum_{\beta}\left|\partial_{X_{1}} s_{\alpha \beta}\right|^{2}+X_{2}^{2} \sum_{\beta}\left|\partial_{X_{2}} s_{\alpha \beta}\right|^{2}\right. \\
& \left.+2 \cos \phi X_{1} X_{2} \sum_{\beta} \operatorname{Re}\left(\partial_{X_{1}} s_{\alpha \beta} \partial_{X_{2}} s_{\alpha \beta}^{\dagger}\right)\right],
\end{aligned}
$$

which agrees ${ }^{22}$ with the result of Ref. 20 .

We now apply our formula Eq. (12) to a one-dimensional quantum structure which is modeled by a double barrier potential $U(x)=X_{1} \delta(x+a)+X_{2} \delta(x-a)$ where $2 a$ is the well width. For this system the Green's function $G\left(x, x^{\prime}\right)$ can be calculated exactly. ${ }^{23}$ With $G\left(x, x^{\prime}\right)$ we can calculate scattering matrix from the Fisher-Lee relation ${ }^{24} s_{\alpha \beta}=-\delta_{\alpha \beta}$ $+i \hbar v G\left(x_{\alpha}, x_{\beta}\right)$, with $v$ the electron velocity in the lead. The adiabatic pump that we consider is operated by changing barrier heights adiabatically and periodically: $X_{1}=V_{10}$ $+V_{p} \sin (\omega t)$ and $X_{2}=V_{20}+V_{p} \sin (\omega t+\phi)$. This can be achieved by microfabricating metallic gates at the barrier region and applying a time dependent gate potential. Since the pumped current is proportional to $\omega^{2}$, we will set $\omega=1$ for convenience. Finally the unit is set by $\hbar=2 m=1 .{ }^{25}$

We first study the pumped heat current with two pumping potentials. Figure 1 depicts the pumped heat current exiting from left lead versus Fermi energy for the symmetric barriers at small pumping amplitude. We have also plotted the transmission coefficient (solid line) versus Fermi energy for comparison. The physical picture of heat flow suggested by Moskalets and Buttiker ${ }^{20}$ is the following: as an electron is scattered by the oscillating barriers or scatterers, the absorption of energy quantum $\hbar \omega$ creates an electron-hole pair. The flow of electron-hole pair leads to the heat transfer. In the symmetric case, we have $I_{q, L}=I_{q, R}$. We see that the heat current is peaked at the resonant levels and is clearly proportional to the density of states of the scattering region. At phase difference $\phi=3 \pi / 4$ (short-dashed line), the line shape of $I_{q}$ is approximately Lorentzian similar to that of transmis- 


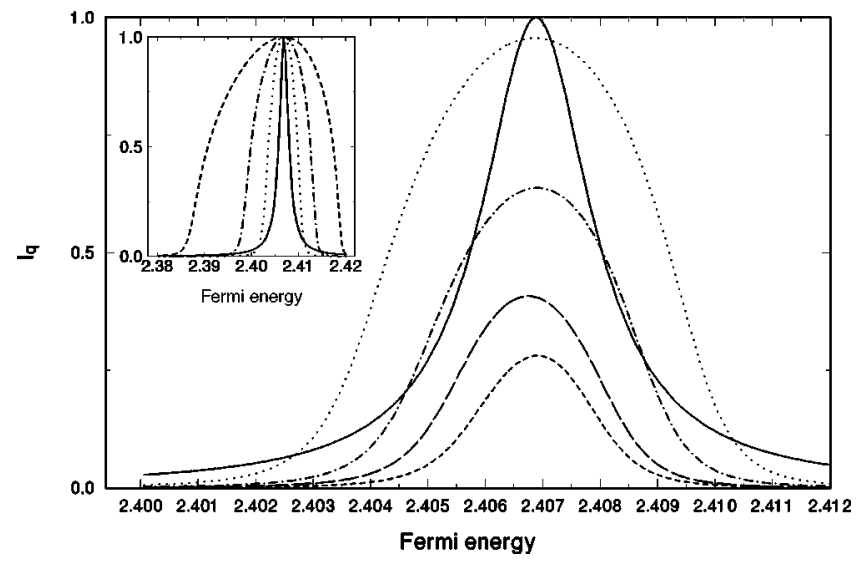

FIG. 1. The transmission coefficient (solid line) and heat current as a function of Fermi energy at different phase differences between two pumping potentials: $\phi=0$ (dotted line), $\phi=\pi / 2$ (dot-dashed line), $\phi=3 \pi / 4$ (short-dashed line). The heat current for a single pump (long-dashed line). For illustrating purpose, the heat current has been multiplied by $10 / 3$. Other parameters, $v_{10}=v_{20}=80$ and $v_{p}=4$. Inset, the heat current as a function of Fermi energy at different pumping amplitudes $v_{p}=5$ (dotted line), $v_{p}=10$ (dotdashed line), $v_{p}=20$ (dashed line). The transmission coefficient is also shown (solid line). The heat current has been normalized to one. The scaling factors are $8 / 3$ for $v_{p}=5,4 / 3$ for $v_{p}=10$, and $2 / 3$ for $v_{p}=20$. Other parameters, $v_{10}=v_{20}=80, \phi=0$.

sion coefficient. As $\phi$ decreases, the line shape is broadened and deviates from Lorentzian considerably. For large pumping amplitude (see inset of Fig. 1), we see that there is significant heat current even in the off resonant case (when Fermi energy is not in line with the resonant level in the static case when pumping potential is off). This is because in the strong pumping regime, the instantaneous resonant level oscillates with a large amplitude and hence can generate heat current in a broad range of energy. Up to the order of $\omega^{2}$, the heat currents $I_{q, L}$ and $I_{q, R}$ are all positive and flow from the scatterers to the reservoir. It is no longer true when highorder frequency contribution is included. Figure 2 shows the heat current as a function of phase difference for different pumping amplitudes. In general, there are two extreme points for the heat current at $\phi=0$ and $\phi=\pi$, where the former corresponds to maximum in heat current and latter to the minimum. In the weak pumping regime, the sinusoidal behavior is seen. In the strong pumping regime, however, we see significant derivation from the sinusoidal behavior. As a result, when increasing $\phi$ from $\phi=0$ to $\pi$, the heat current drops not as fast as in the weak pumping regime away from $\phi=\pi$. However, near $\phi=\pi$, the heat current decreases much faster. Figure 3 displays the heat current as a function of pumping amplitude. The general behaviors of heat current at different $\phi$ are similar. We see that initially, the heat current increases quadratically with pumping amplitude in the weak pumping regime and then quickly approaches the linear regime in the strong pumping regime. This behavior persists for single pumping potential. In the inset, we present the result for asymmetric barriers. Here $v_{10}=4, v_{20}=6$, and $v_{p}=0.5, I_{q, L}$ is about twice as large as $I_{q, R}$. This is reasonable because it is easier for heat current to tunnel through the

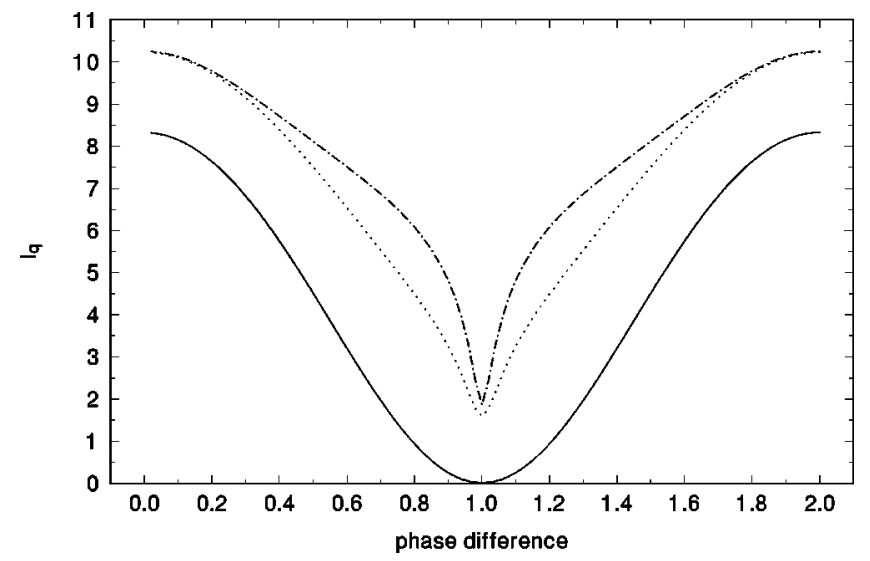

FIG. 2. The heat current as a function of phase differences $\phi / \pi$ at different pumping amplitudes: $v_{p}=1$ (solid line), $v_{p}=20$ (dotted line), $v_{p}=40$ (dot-dashed line). The heat current for a single pump (long-dashed line). For illustrating purpose, the heat current has been multiplied by a factor of 200,20/3,10/3, respectively for $v_{p}$ $=1,20$, 40. Other parameters, $v_{10}=v_{20}=80$ and $E_{F}=2.4069$.

lower barrier. In general, the electric pumped current is linear in frequency for two pumping potentials whereas for single pumping potential, it must be zero up to the first order in frequency. 8,16 The heat current is different. For both single pump and two potential pump, the heat currents are of order $\omega^{2}$. In the weak pumping limit, there is a simple relationship between the following three heat currents: ${ }^{20} I_{\pi / 2}=I_{L}+I_{R}$, where $I_{\pi / 2}$ is the heat current of two pumping potentials with phase difference $\phi=\pi / 2, I_{L}$ is the heat current of single pump due to the left oscillating barrier, and $I_{R}$ heat is the current due to the right barrier. In the strong pumping regime, the scattering matrix depends on both $X_{1}$ and $X_{2}$ in a nonlinear fashion, this simple relation is no longer valid. Denoting the ratio $\tau \equiv I_{\pi / 2} /\left(I_{L}+I_{R}\right)$, we found that at small pumping amplitude, $\tau \sim 1$. As one increases the pumping amplitude the ratio $\tau$ decreases and quickly saturates around

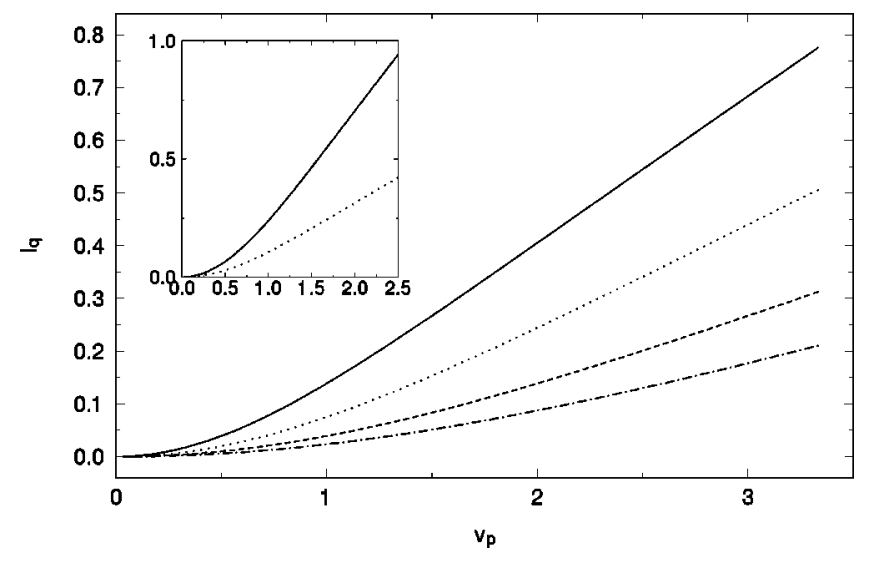

FIG. 3. The heat current as a function of pumping amplitude at different phase difference: $\phi=0$ (solid line), $\phi=\pi / 2$ (dotted line), $\phi=3 \pi / 4$ (dotted-dash line). The heat current for a single pump (dashed line). The heat current has been multiplied by a factor of $10 / 3$. Other parameters are the same as the inset of Fig. 2. Inset, $I_{q, L}$ (solid line) and $I_{q, R}$ (dotted line) vs pumping amplitude for asymmetric barriers. Here $\phi=0$ and $E_{F}=1.65$. The scaling factor is $1 / 3$. 


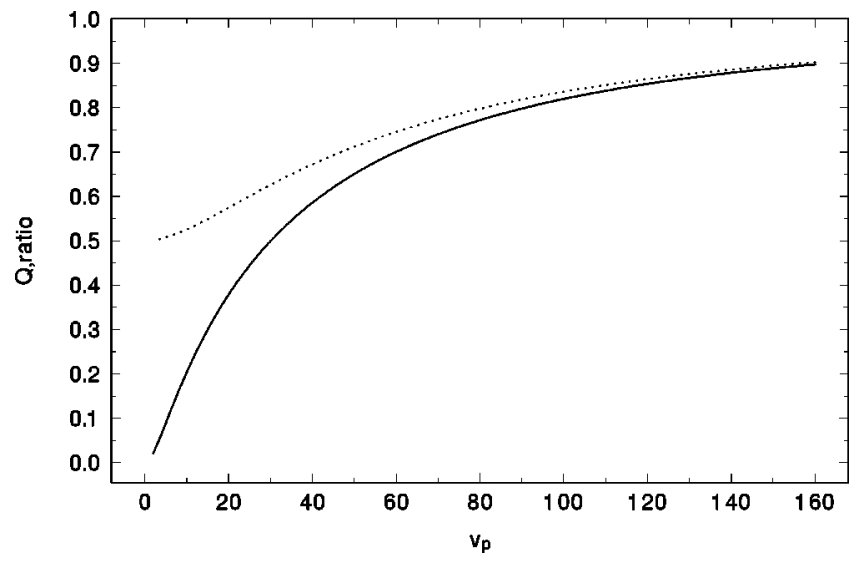

FIG. 4. The pumped charge per cycle (solid line), the ratio between the power of Joule heat and the heat current (dotted line) as a function of pumping amplitude. The system parameters are $v_{10}$ $=v_{20}=80, \phi=3 \pi / 4$, and $E_{F}=2.4069$.

$\tau \sim 0.8$. This is understandable since the behavior of $I_{\pi / 2}$, $I_{L}$, and $I_{R}$ is similar as one increases the pumping amplitude, one obtains the nearly constant ratio in the linear pumping amplitude dependence regime (see Fig. 3).

Finally, we study the dissipation of the pump by comparing the heat current and the power of Joule heat produced by the electric current. ${ }^{26}$ We found that the heat current is always larger than the corresponding power of Joule heat as predicted by Ref. 19. In Fig. 4, we plot the pumped charge per cycle and the ratio between the power of Joule heat and the heat current as a function of pumping amplitude $v_{p}$. We see that the pumped charge increases as $v_{p}$ increases. In the large $v_{p}$ limit, the pumped charge will eventually reach the maximum value $Q=e{ }^{14,17}$ We see from Fig. 4 that the ratio also approaches to one just like the pumped charge. Therefore, we conclude that the double barrier pump we studied can be optimal in very strong pumping limit.

In summary, we have developed a general theory for the pumped heat current at finite pumping amplitude and frequency which allows us to investigate the pumped heat current in both weak and strong pumping regimes as different system parameters vary. As the pumping amplitude varies, we observed the crossover of pumped heat current from quadratic dependence in the weak pumping regime to linear dependence in the strong pumping regime. In the strong pumping regime, the pumped heat current shows strong nonlineality as a function of phase difference between two pumping potentials. For the single pump, we found that the amplitude of pumped heat current is of the same order as that of two pumping potentials. Finally, our numerical results show that the double barrier structure we examined can be an optimal pump in the strong pumping regime. Due to the perturbative nature of our approach, it is very difficult to calculate the pumped heat current for general frequency in the strong pumping regime. However, it is possible to solve this problem using the scattering matrix approach by Wagner. ${ }^{27}$

We gratefully acknowledge support by a RGC grant from the SAR Government of Hong Kong under Grant No. HKU 7091/01P and a CRCG grant from the University of Hong Kong.
*Electronic mail: jianwang@hkusub.hku.hk

${ }^{1}$ P.W. Brouwer, Phys. Rev. B 58, R10 135 (1998).

${ }^{2}$ M. Switkes, C. Marcus, K. Capman, and A.C. Gossard, Science 283, 1905 (1999).

${ }^{3}$ F. Zhou, B. Spivak, and B.L. Altshuler, Phys. Rev. Lett. 82, 608 (1999).

${ }^{4}$ M. Wagner, Phys. Rev. Lett. 85, 174 (2000).

${ }^{5}$ J.E. Avron, A. Elgart, G.M. Graf, and L. Sadun, Phys. Rev. B 62, R10 618 (2000).

${ }^{6}$ I.L. Aleiner, B.L. Altshuler, and A. Kamenev, Phys. Rev. B 62, 10373 (2000).

${ }^{7}$ Y.D. Wei, J. Wang, and H. Guo, Phys. Rev. B 62, 9947 (2000); Y.D. Wei, J. Wang, H. Guo, and C. Roland, ibid. 64, 075304 (2001); J. Wang, Y.D. Wei, B.G. Wang, and H. Guo, Appl. Phys. Lett. 79, 3977 (2001).

${ }^{8}$ M.G. Vavilov, V. Ambegaokar, and I.L. Aleiner, Phys. Rev. B 63, 195313 (2001)

${ }^{9}$ P.W. Brouwer, Phys. Rev. B 63, 121303 (2001); M.L. Polianski and P.W. Brouwer, ibid. 64, 075304 (2001).

${ }^{10}$ P. Sharma and C. Chamon, Phys. Rev. Lett. 87, 096401 (2001).

${ }^{11}$ X.B. Wang and V.E. Kravtsov, Phys. Rev. B 64, 033313 (2001).

${ }^{12}$ I.L. Aleiner and A.V. Andreev, Phys. Rev. Lett. 81, 1286 (1998).

${ }^{13}$ T.A. Shutenko, I.L. Aleiner, and B.L. Altshuler, Phys. Rev. B 61, 10366 (2000).

${ }^{14}$ Y. Levinson, O. Entin-Wohlman, and P. Wolfle, Physica A 302, 335 (2001).
${ }^{15}$ M. Moskalets and M. Buttiker, Phys. Rev. B 64, 201305 (2001).

${ }^{16}$ B.G. Wang, J. Wang, and H. Guo, Phys. Rev. B 65, 073306 (2002).

${ }^{17}$ J. Wang and B.G. Wang, Phys. Rev. B 65, 153311 (2002).

${ }^{18}$ B.G. Wang and J. Wang, Phys. Rev. B 65, 233319 (2002).

${ }^{19}$ J.E. Avron, A. Elgart, G.M. Graf, and L. Sadun, Phys. Rev. Lett. 87, 236601 (2001).

${ }^{20}$ M. Moskalets and M. Buttiker, Phys. Rev. B 66, 035306 (2002).

${ }^{21}$ A. Alekseev, cond-mat/0201474 (unpublished).

${ }^{22}$ The difference between our Eq. (11) and Eq. (21) in Ref. 20 is due to the fact that the scattering matrix and hence $T_{ \pm \omega}$ defined in Ref. 20 is energy independent. As a result, a term $\omega^{2}(E$ $\left.-E_{F}\right) \partial_{E} f\left(\partial_{E} T_{-\omega}+\partial_{E} T_{+\omega}\right)$ is missing in Eq. (21) of Ref. 20. This can be easily understood from Eq. (6) of Ref. 16. Finally, in order to compare our result with that of Ref. 20 one should multiply 1/4 in Eqs. (21) and (39) of Ref. 20.

${ }^{23}$ M.K. Yip, J. Wang, and H. Guo, Z. Phys. B: Condens. Matter 104, 463 (1997).

${ }^{24}$ D.S. Fisher and P.A. Lee, Phys. Rev. B 23, 6851 (1981).

${ }^{25}$ For the GaAs system with $a=1000 A$, the energy unit is $E$ $=56 \mu \mathrm{eV}$.

${ }^{26}$ The power of Joule heat is defined as $\left(\pi / 2 T_{p}\right) \int_{0}^{T_{p}} d t(d Q / d t)^{2}$ where $Q=\int_{0}^{T_{p}} d t(d Q / d t)$ gives the pumped charge and $I_{p}$ $=Q / T_{p}$ the pumped electric current.

${ }^{27}$ M. Wagner, Phys. Rev. A 51, 798 (1995). 